\title{
Pharmacodynamic Analysis of Magnetic Resonance Imaging-Monitored Focused Ultrasound-Induced Blood-Brain Barrier Opening for Drug Delivery to Brain Tumors
}

\author{
Po-Chun Chu, ${ }^{1}$ Wen-Yen Chai, ${ }^{1,2}$ Han-Yi Hsieh, ${ }^{1}$ Jiun-Jie Wang, ${ }^{3}$ Shiaw-Pyng Wey, ${ }^{3}$ \\ Chiung-Yin Huang, ${ }^{4}$ Kuo-Chen Wei, ${ }^{4}$ and Hao-Li Liu ${ }^{1}$ \\ ${ }^{1}$ Department of Electrical Engineering, Chang-Gung University, 259 Wen-Hwa 1st Road, Kwei-Shan, Tao-Yuan 333, Taiwan \\ ${ }^{2}$ Department of Diagnostic Radiology, Chang-Gung University and Memorial Hospital, 5 Fu-shin Street, Kwei-Shan, \\ Tao-Yuan 333, Taiwan \\ ${ }^{3}$ Department of Medical Imaging and Radiological Sciences, Chang-Gung University, 259 Wen-Hwa 1st Road, Kwei-Shan, \\ Tao-Yuan 333, Taiwan \\ ${ }^{4}$ Department of Neurosurgery, Chang-Gung Memorial Hospital, 5 Fu-shin Street, Kwei-Shan, Tao-Yuan 333, Taiwan
}

Correspondence should be addressed to Hao-Li Liu; haoliliu@mail.cgu.edu.tw

Received 21 January 2013; Accepted 25 February 2013

Academic Editor: Fan-Lin Kong

Copyright (C) 2013 Po-Chun Chu et al. This is an open access article distributed under the Creative Commons Attribution License, which permits unrestricted use, distribution, and reproduction in any medium, provided the original work is properly cited.

\begin{abstract}
Microbubble-enhanced focused ultrasound (FUS) can enhance the delivery of therapeutic agents into the brain for brain tumor treatment. The purpose of this study was to investigate the influence of brain tumor conditions on the distribution and dynamics of small molecule leakage into targeted regions of the brain after FUS-BBB opening. A total of 34 animals were used, and the process was monitored by 7T-MRI. Evans blue (EB) dye as well as Gd-DTPA served as small molecule substitutes for evaluation of drug behavior. EB was quantified spectrophotometrically. Spin-spin $\left(R_{1}\right)$ relaxometry and area under curve (AUC) were measured by MRI to quantify Gd-DTPA. We found that FUS-BBB opening provided a more significant increase in permeability with small tumors. In contrast, accumulation was much higher in large tumors, independent of FUS. The AUC values of Gd-DTPA were well correlated with EB delivery, suggesting that Gd-DTPA was a good indicator of total small-molecule accumulation in the target region. The peripheral regions of large tumors exhibited similar dynamics of small-molecule leakage after FUS-BBB opening as small tumors, suggesting that FUS-BBB opening may have the most significant permeability-enhancing effect on tumor peripheral. This study provides useful information toward designing an optimized FUS-BBB opening strategy to deliver small-molecule therapeutic agents into brain tumors.
\end{abstract}

\section{Introduction}

Focused ultrasound beams (FUS) in the presence of circulating microbubbles can temporarily open the bloodbrain barrier (BBB opening) of capillaries in the central nervous system (CNS) parenchyma [1-3]. Bursts of acoustic ultrasound induce microbubble cavitation in the vasculature, and the resultant shear stress temporarily disrupts tight junctions to enhance blood-brain permeability. This BBBopening process can be carried out at moderate acoustic pressures to minimize adverse effects on vascularture and prevent damage to neurons [3-7], while facilitating localized delivery of chemotherapeutic agents from the vasculature to the pathological brain parenchyma and CNS [8-11]. Since more than $95 \%$ of the therapeutic agent normally cannot penetrate CNS tight junctions [12], this novel approach provides a unique opportunity for local delivery of therapeutic agents across the $\mathrm{BBB}$ and into the targeted site, thus opening a new frontier of CNS drug delivery.

Brain tumors could potentially be treated by FUS-BBB opening to enhance chemotherapeutic agent delivery. In the United States, at least 18,000 patients are diagnosed with glioblastoma multiforme (GBM) each year, comprising more than half of the malignant primary brain tumors [13], 


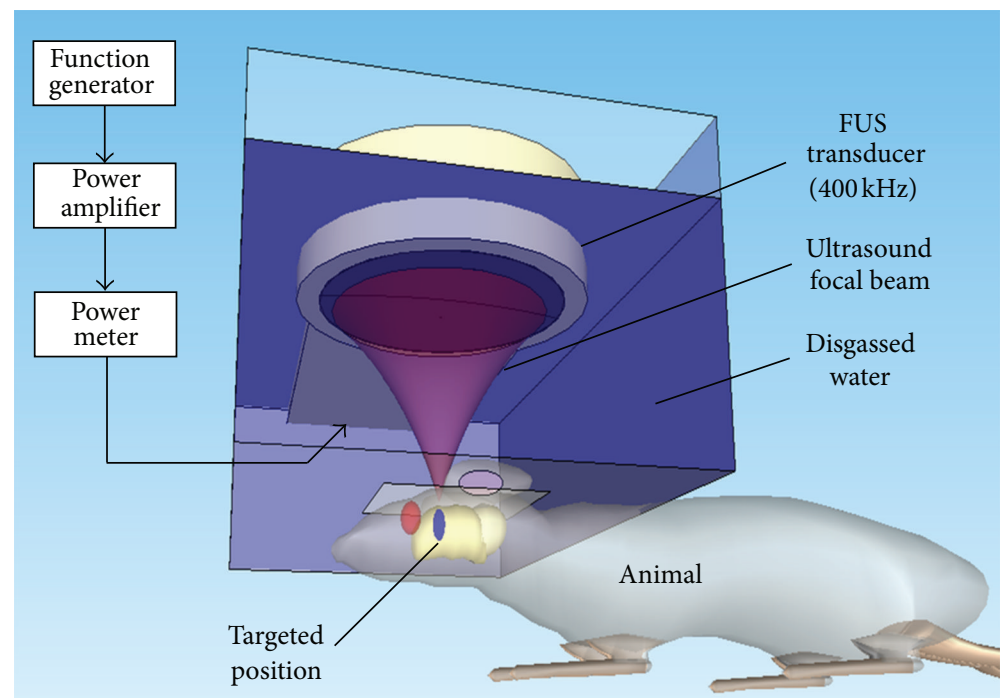

FIGURE 1: Schematic showing the experimental setup of the focused ultrasound exposure system.

and chemotherapy is an important treatment modality [14]. Recent preclinical studies showed that FUS-BBB opening can effectively enhance local deposition and concentration of chemotherapeutics including BCNU [11], liposomal doxorubicin $[9,15]$, and chemodrugs carried by novel nanocarriers [16].

Currently, FUS-mediated CNS drug delivery is monitored by magnetic resonance imaging (MRI) by intravenous (IV) injection of gadolinium diethylenetriamine penta-acetic acid (Gd-DTPA) contrast agent together with chemotherapeutic drug. The MRI signal intensity increase caused by the leakage of Gd-DTPA thus serves as an indicator to estimate drug concentration $[4,8,9,17]$. Most studies have focused on analyzing the effects of FUS exposure parameters such as acoustic pressure amplitude, ultrasound frequency, pulse length, pulse repetition frequency, exposure duration, and microbubble dose on BBB opening [5, 18-23]. However, pharmacodynamic analysis including the dynamics and distribution of the specific molecular agent in the brain is critical for evaluating specific drug delivery. MRI could also be used for pharmacological endpoint evaluation using concurrently administered MR contrast agents as surrogate indicators of therapeutic drug concentrations. Although the kinetics of contrast agent permeability of a defective bloodbrain barrier have been measured using MR compartment modeling [24-26], these studies were performed in normal animal brains, and so far the detailed pharmacodynamic behavior of contrast agents after FUS-BBB opening remains uncertain in brain tumors.

The purpose of this study was to conduct an MRI pharmacodynamic analysis of FUS-BBB opening in brain tumors in an animal model. Injected Gd-DTPA contrast agent was used to characterize pharmacodynamic changes as a function of time after BBB opening, in both normal and brain-glioma animals. We also attempted to establish the correlation between deposition of Gd-DTPA by in vivo semiquantification and the quantitation of another surrogate,
Evans blue dye, by spectrophotometry after sacrifice. Finally, we evaluated the pharmacodynamic changes affected by FUS$\mathrm{BBB}$ opening in various grades of gliomas.

\section{Methods}

2.1. FUS Setup. A focused ultrasound transducer was used to generate ultrasound focal energy (IMASONIC, France; diameter $=60 \mathrm{~mm}$, radius of curvature $=80 \mathrm{~mm}$, frequency $=$ $400 \mathrm{kHz}$, and electric-to-acoustic efficiency $=70 \%$ ) (Figure 1). An arbitrary function generator (33120A, Agilent, Palo Alto, CA and DS345, Stanford Research Systems, Sunnyvale, CA) was used to generate the driving signal, which was then fed into a radiofrequency power amplifier (150A100B, Amplifier Research, Souderton, PA). The focal zone distribution of the intensity of the ultrasound field was measured in an acrylic water tank filled with deionized, degassed water. The measured diameter of the half-maximum pressure amplitude was $2 \mathrm{~mm}$, and the length of the produced focal zone was $15 \mathrm{~mm}$. Animals underwent isofluorane anesthesia before ultrasound treatment. The animal was laid prone and placed directly under an acrylic water tank (with a window of $4 \times 4 \mathrm{~cm}^{2}$ at its bottom sealed with a thin film to allow entry of the ultrasound energy), using ultrasound gel to fill the interspaces between the animal head and the thinfilm window. SonoVue SF6-coated ultrasound microbubbles $(2-5 \mu \mathrm{m}$ mean diameter, $2.4 \mu \mathrm{L} / \mathrm{kg}$; Bracco Diagnostics Inc.) were IV administered by burst injection with $0.1 \mathrm{~mL}$ of saline solution containing $0.01 \mathrm{~mL}$ heparin. After injecting the microbubbles, burst-tone mode ultrasound at a pressure of $0.4 \mathrm{MPa}$ (peak negative value; measured in the free-field) was delivered to the brain with the center of the focal zone positioned at a penetration depth of 4$5 \mathrm{~mm}$ under the scalp (burst length $=10 \mathrm{~ms}$, pulse repetition frequency $=1 \mathrm{~Hz}$, and total sonication duration = $90 \mathrm{~s})$. 


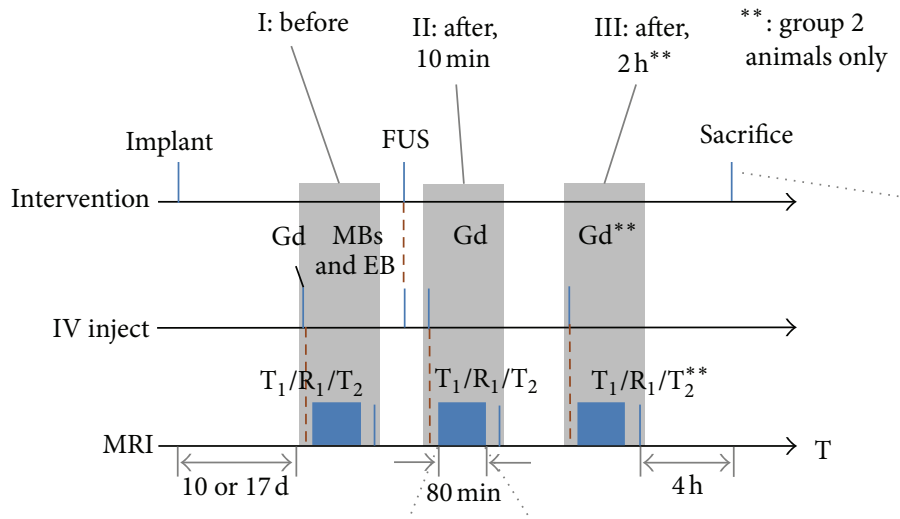

(a)

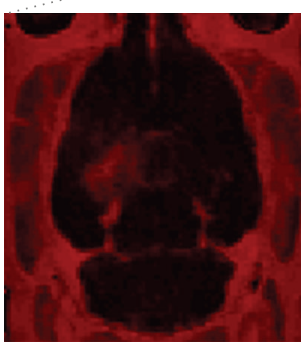

$10 \mathrm{~min}$

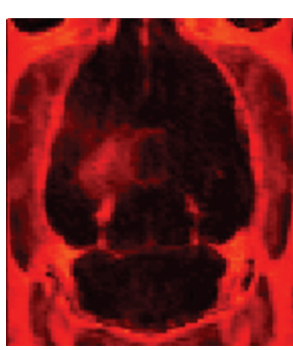

20 min

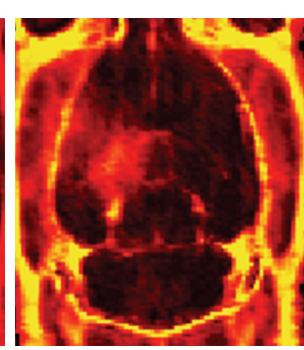

40 min

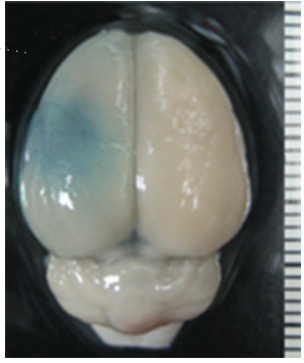

(b)

(c)

FIgURE 2: (a) Experimental protocol for 1st- and 2nd-group animal experiments. MRI images were acquired in time slots I/II for group 1 animals and in time slots I/II/III for group 2 animals; double asterisks $\left({ }^{* *}\right)$ indicate group- 2 experiments only. (b) FUS-exposed brain of a normal animal with Evans blue extravasations to identify the location of BBB opening. (c) $\mathrm{R}_{1}$ accumulating map showing Gd-DTPA accumulation in the BBB-opening location of a normal animal over time.

2.2. Animal Experiment Design. All animal experiments were approved by the Institutional Animal Care and Use Committee of Chang Gung University and adhered to the experimental animal care guidelines. A total of 34 animals (male Sprague-Dawley rats (250-300 g)) were used, including normal $(n=18)$ and tumor animals $(n=16)$. Experiments were divided into two groups. In group 1 , the aim was to confirm the correlation between Gd-DTPA leakage (concentration measured by relaxometry) and Evans blue (EB) dye (concentration measured spectrophotometrically) after FUS-BBB treatment. Subgroups included (1) normal rats $(n=18)$ and (2) tumor rats $(n=4)$, and the first subgroup underwent FUS-BBB opening. Subgroups were confirmed by dynamic contrast-enhanced (DCE) MRI with Gd-DTPA (molecular weight $=938 \mathrm{Da})$. In addition, EB dye (molecular weight $=960 \mathrm{Da})$ was IV injected into the animals, and the amount of EB deposited in the brain was quantified spectrophotometrically (procedure described below). In the first subgroup of group 1 , contrast-enhanced $\mathrm{T}_{1}$-weighted imaging was first performed to estimate Gd-DTPA concentration after $\mathrm{BBB}$ opening, followed by $\mathrm{T}_{2}$-weighted imaging to provide a reference of tumor morphology. The second subgroup underwent the same scanning process without FUS induced.
In experimental group 2, our aim was to monitor the increase in Gd-DTPA accumulation in tumor-bearing animals after conducting FUS-BBB opening. Animals were divided into two subgroups: (1) animals receiving FUS exposure 10 days after tumor implantation (tumor volume typically $\left.<0.05 \mathrm{~cm}^{3}\right)$ with FUS-BBB opening $(n=6)$ and (2) animals receiving FUS exposure 17 days after tumor implantation (tumor volume typically $>0.05 \mathrm{~cm}^{3}$ ) with FUSBBB opening $(n=6)$. Tumor volume was measured by $\mathrm{T}_{2}$-weighted MRI. In group 2, animals were subjected to three 80-minute-long MR relaxometry-based imaging sessions (before FUS exposure, and 10-min and 120-min after FUS exposure). Detailed experimental procedures are shown in Figure 2.

2.3. Rat Brain Glioma Model. C6 glioma cells were harvested by trypsinization and cultured at a concentration of $1 \times$ $10^{5}$ cells $/ \mathrm{mL}$ for implantation. For intracranial injection into the striatum of rat brains, cells were washed once with phosphate buffered saline (PBS). Male Sprague-Dawley rats $(250-300 \mathrm{~g})$ were anesthetized by intraperitoneal administration of ketamine $(100 \mathrm{mg} / \mathrm{kg})$ and immobilized on a stereotactic frame. A sagittal incision was made through the 
skin overlying the calvarium, and a small dental drill was used to make a hole in the exposed cranium, $0.5 \mathrm{~mm}$ anterior and $3 \mathrm{~mm}$ lateral to the bregma on the left side of skull. C6 cell suspension $(5 \mathrm{~mL})$ was injected at a depth of $4.5 \mathrm{~mm}$ from the brain surface. The injection was performed over a 10 minute period, and the needle was withdrawn over another 2 minutes. Ten days after implantation, tumor sizes were measured by MRI.

2.4. Spectrophotometric Quantitation of Evans Blue Dye. EB dye (3\% in saline) was IV injected $(2 \mathrm{mg} / \mathrm{kg})$, and the animals were sacrificed two hours later. All animals were first deeply anesthetized with $10 \%$ chloral hydrate and infused with heparinized saline through the cardiac ventricle until colorless infusion fluid was obtained from the atrium. After the rats had been sacrificed by decapitation, the hemispheres of the brain were separated along the transverse suture. Then both hemispheres were weighed and placed in formamide $(1 \mathrm{~mL} / 100 \mathrm{mg})$ at $60^{\circ} \mathrm{C}$ for $24 \mathrm{~h}$. The sample was centrifuged for $20 \mathrm{mins}$ at $14,000 \mathrm{rpm}$. The concentration of dye extracted from each brain was determined spectrophotometrically at $620 \mathrm{~nm}$ and was compared with a standard graph created by recording optical densities from serial dilutions of EB in $0.9 \%$ sodium chloride solution. The EB tissue content was quantified using a linear regression standard curve derived from seven concentrations of the dye.

2.5. MRI. For in vitro measurements, Gd-DTPA (Omniscan, $0.3 \mathrm{~mL} / \mathrm{kg}$, Magnevist) was diluted with physiological saline to $0.12,0.24,0.49,0.97,1.96$, and $3.9 \mu \mathrm{M}$. Circular wells (inner diameter $=5 \mathrm{~mm}$ ) were filled with $200 \lambda$ of contrast agent sample or physiological saline as control and were placed in the MR scanner (Clinscan, Bruker, Germany; 7 Tesla). Spin-lattice relaxivity maps were calculated from two $\mathrm{T}_{1}$ weighted images with different flip angles (gradient recalled echo sequence, $\mathrm{TR} / \mathrm{TE}=2.3 \mathrm{~ms} / 0.76 \mathrm{~ms}$, slide thickness = $0.8 \mathrm{~mm}$, matrix $=132 \times 192$, and flip angle $\left.=5^{\circ} / 20^{\circ}\right)$. The correlation between $\mathrm{R}_{1}\left(=1 / \mathrm{T}_{1}\right)$ mapping and Gd-DTPA concentration was determined [27].

In the animal experimental group, FUS-induced BBB opening was monitored by MRI with a 7-Tesla magnetic resonance scanner (Bruker ClinScan, Germany) and a 4-channel surface coil. The mouse was placed in an acrylic holder, positioned in the center of the magnet, and anesthetized with isoflurane gas (1-2\%) at 50-70 breaths/min during the entire MRI procedure.

In the first experimental group, the distribution and dynamics of Gd-DTPA leakage were investigated immediately after conducting FUS-BBB opening. After FUSBBB opening, animals were immediately relocated into the MR scanning room, and contrast-enhanced $\mathrm{T}_{1}$-weighted images with different flip angles were acquired to calculate spin-lattice relaxivity maps by transferring two images with different flip angles (gradient recalled echo sequence, $\mathrm{TR} / \mathrm{TE}=2.3 \mathrm{~ms} / 0.76 \mathrm{~ms}$, slice thickness $=0.8 \mathrm{~mm}$, slice number $=14$, matrix $=132 \times 192$, and flip angle $=5^{\circ} / 20^{\circ}$ ) Images were sequentially acquired over $80 \mathrm{~min}$ with a time interval of 60 seconds for area under the curve (AUC) calculation. Upon completion of the 10th acquisition, a diluted bolus of Gd-DTPA was IV injected through a catheter at an infusion rate of $6 \mathrm{~mL} / \mathrm{s}$. In the second experimental group, three sets of Gd-DTPA-leakage distribution/dynamics were investigated, including (I) before FUS exposure, (II) immediately after FUS exposure, and (III) two hours after FUS exposure. Immediately after conducting FUS-BBB opening, turbo spin echo (TSE) $\mathrm{T}_{2}$ weighted images were obtained as a reference to identify the tumor region (repetition time (TR)/echo time (TE) = $2540 / 41 \mathrm{~ms}, \mathrm{FOV}=34 \times 40 \mathrm{~mm}^{2}$, in-plane resolution $=$ $0.4 \times 0.3 \mathrm{~mm}^{2}$, and slice thickness $=0.6 \mathrm{~mm}$ ).

2.6. MR Analysis of Gd-DTPA Accumulation and Distribution after FUS-BBB Opening. In $\mathrm{R}_{1}$-map analysis, a region of interest (ROI) was selected and compared with the nonenhanced contralateral brain to determine the increase in Gd-DTPA concentration caused by BBB opening. AUC maps were then transferred from a series of time-dependent $R_{1}$ maps (up to $80 \mathrm{~min}$ ) to determine pharmacodynamic characteristics of Gd-DTPA for comparison with the dynamics of EB dye permeability. Thus, the total area (AUC) is given by the following equation:

$$
\mathrm{AUC}_{80 \min }=\frac{\int C p t \cdot d t}{V}
$$

where Cpt are vertical segments under the Gd-DTPA concentration curve area and $V$ is total ROI volume.

In experimental group 2, ROIs were selected in the targeted tumor area which was based on the tumor dimensions defined in $\mathrm{T}_{2}$ images (the same ROI as in the contralateral brain was selected). The distribution and dynamics of GdDTPA leakage were evaluated for different tumor sizes including 10 days after implantation (typically $<0.05 \mathrm{~cm}^{3}$ ) and 17 days after implantation (typically $>0.05 \mathrm{~cm}^{3}$ ) and were divided by the tumor dimension. ROI including the entire tumor and the contralateral area were selected. Moreover, in order to evaluate the homogeneity of Gd-DTPA leakage, tumors with dimension $>0.05 \mathrm{~cm}^{3}$ were further divided into tumor core (inner half of the area) and tumor peripheral (outer half of the area), based on $\mathrm{T}_{2}$ images.

2.7. Histology. Albumin-bound EB dye was IV injected as a bolus immediately after sonication. BBB opening was quantified as extravasation of EB. Tumor model animals were sacrificed about $2 \mathrm{~h}$ after sonication and MR scanning. Brain samples were serially sectioned ( $2 \mu \mathrm{m}$ thickness) using the same slice direction as in MRI analysis. Representative sections were stained with hematoxylin and eosin (HE). Tumor morphology was histologically evaluated.

\section{Results}

$\mathrm{BBB}$ opening was clearly evidenced by staining with EB dye. A typical image of a normal BBB-opened brain stained with EB dye is shown in Figure 2(b). In addition, a series 

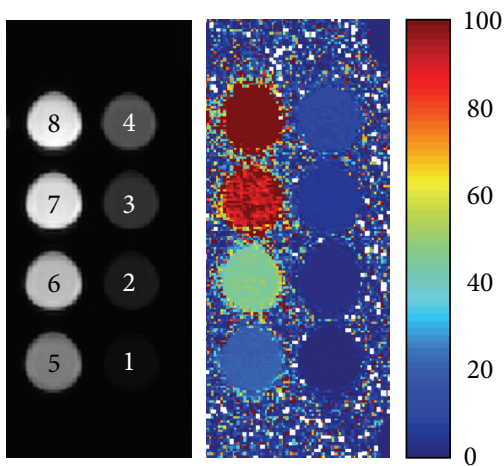

(a)

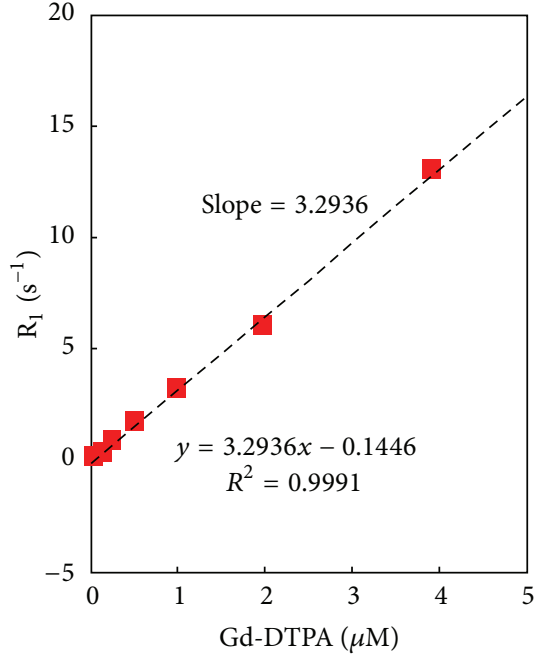

(b)

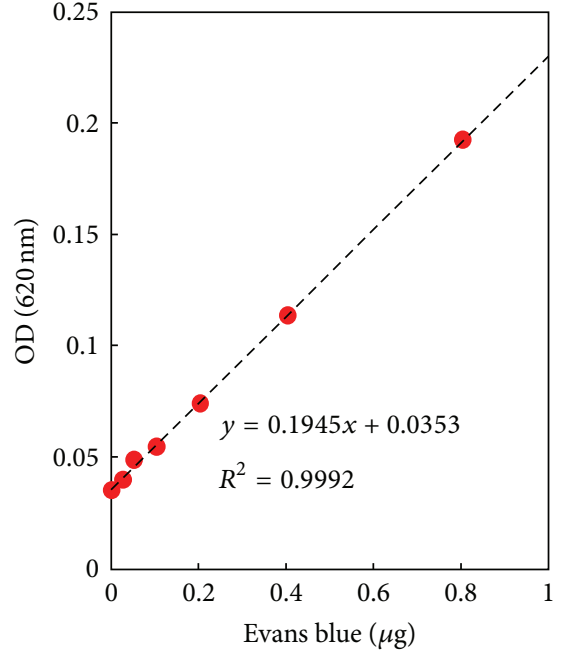

(c)

FIGURE 3: (a) $\mathrm{T}_{1}$ image and corresponding $\mathrm{R}_{1}$ map for the in vitro Gd-DTPA phantom at increasing concentrations ( 1 and 2: water; 3: $0.12 \mu \mathrm{M}$; 4: $0.24 \mu \mathrm{M} ; 5: 0.49 \mu \mathrm{M} ; 6: 0.97 \mu \mathrm{M} ; 7: 1.96 \mu \mathrm{M} ; 8: 3.9 \mu \mathrm{M}$ ). (b) Dependence of $\mathrm{R}_{1}$ on Gd-DTPA concentration; relaxivity was estimated as about $3.2936 \mathrm{~s}^{-1} \mathrm{mM}^{-1}$. (c) Calibration curve of spectrophotometrically determined EB concentration. EB concentrations ranging from 0 to $0.8 \mu \mathrm{g}$ were tested, resulting in O.D. readings of 0.035 to 0.19 at $620 \mathrm{~nm}$.

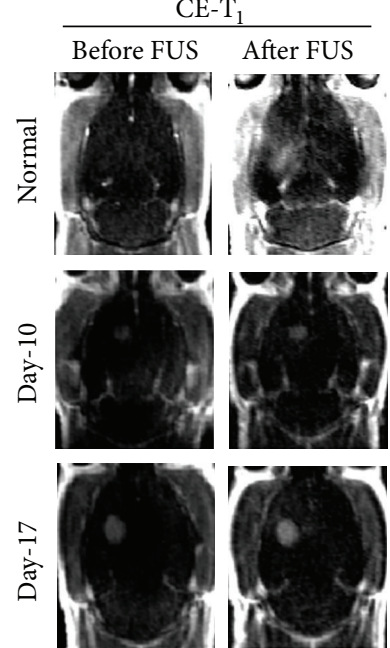

(a)

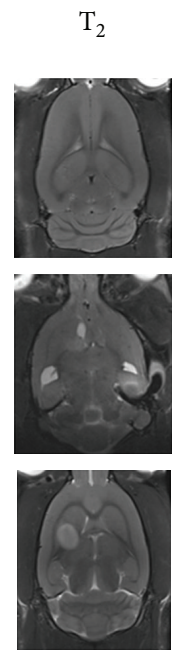

(b)

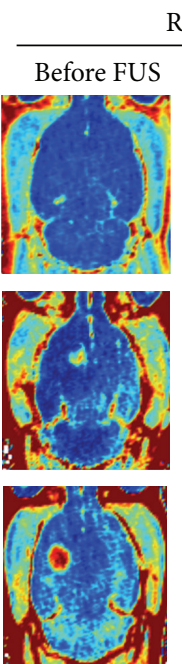

(c)
$\mathrm{R}_{1} \quad \mathrm{AUC}_{80 \text { min }}$

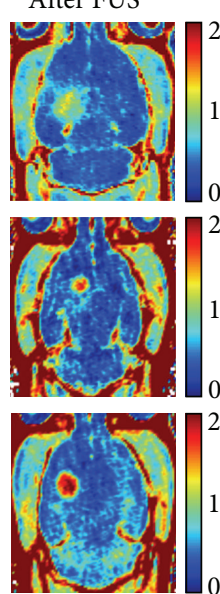

(d)

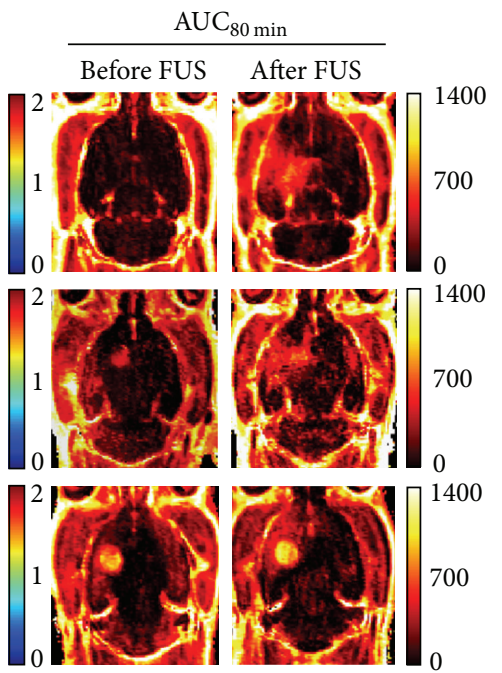

FIgURE 4: Typical MRI for normal animals (upper) as well as animals after 10-day (middle) and 17-day (bottom) tumor implantations. (a) Contrast-enhanced $\mathrm{T}_{1}$ images before and after FUS exposure. (b) $\mathrm{T}_{2}$ images (after FUS). (c) $\mathrm{R}_{1}$ maps before and after FUS exposure. (d) Area under the $\mathrm{R}_{1}$ curve over 80 minutes (denoted as $\mathrm{AUC}_{80 \text { min }}$ ) before and after FUS exposure.

of $\mathrm{R}_{1}$ maps obtained at different time points after FUS$\mathrm{BBB}$ opening demonstrated the dynamic change in GdDTPA accumulation in a normal brain, with particularly high leakage at the sonication site (Figure 2(c)).

$\mathrm{R}_{1}$ relaxivity of Gd-DTPA and ELISA measurements of EB dye concentration were calibrated in vitro. The detected $\mathrm{R}_{1}$-signal increased in a highly linear manner with Gd-DTPA concentration (input concentrations of $0,0.25,0.5,1,2$, and $4 \mu \mathrm{M})$ as shown by the calibration curve $\left(r^{2}=0.9991\right)$ (Figure 3(b)). The relaxivity of Gd-DTPA contrast agent was found to be 3.3 at 7 Tesla. The detected ELISA signal also increased in a highly linear manner with $\mathrm{EB}$ concentration (Figure 3(c); $\left.r^{2}=0.9992\right)$. These calibration curves thus allowed precise quantitation of Gd-DTPA and EB deposition in the brain.

FUS-induced BBB opening was verified by CE-MRI. Typical CE- $T_{1}$ images, $T_{2}$ images, $R_{1}$ maps, and AUC maps in normal, 10-day glioma, and 17-day glioma animals are shown in Figure 4. In normal animals, the BBB-opened area was clearly visible in $\mathrm{T}_{1}$-weighted images. $\mathrm{T}_{2}$-weighted images did not show any evidence of FUS-induced damage at the target location at pressure amplitudes of $0.4 \mathrm{MPa}$ (Figure 4 ). In the 


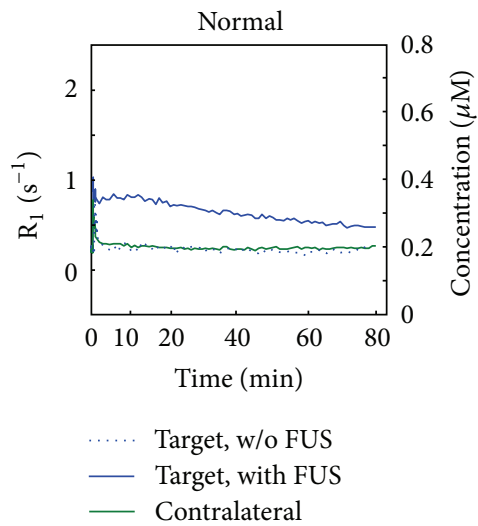

(a)

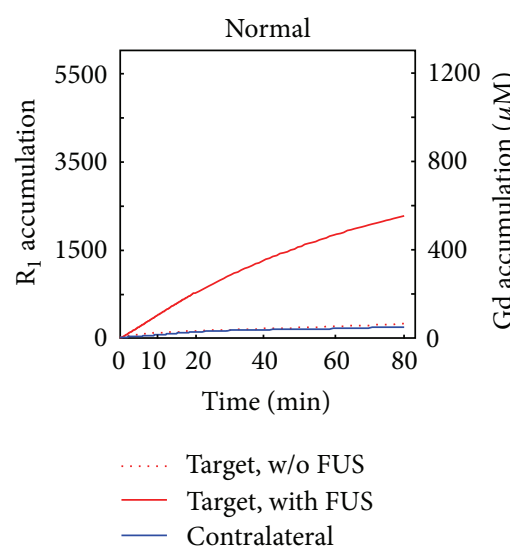

(d)

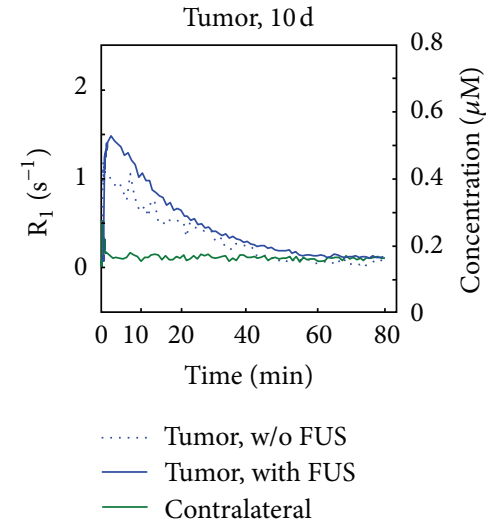

(b)

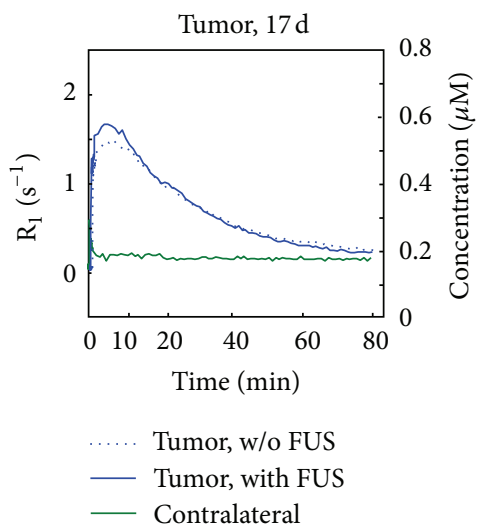

(c)

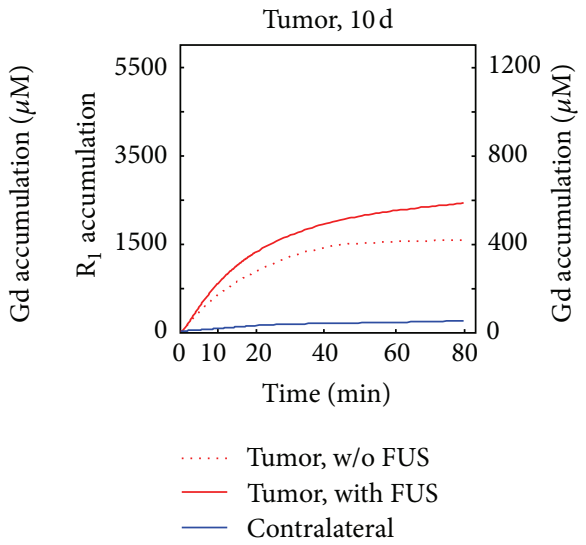

(e)

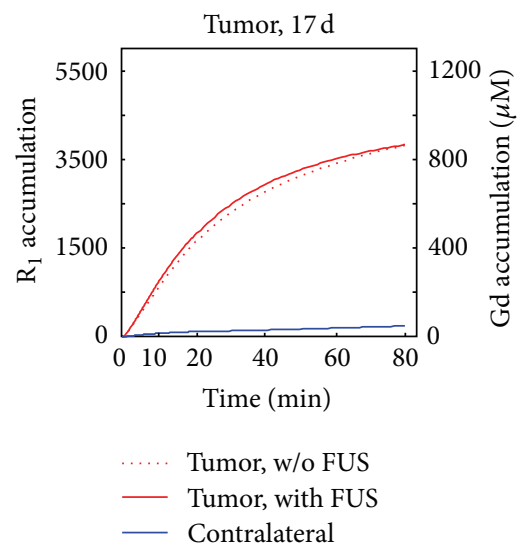

(f)

FIgURE 5: (a)-(c) $R_{1}$ change as a function of time before and after FUS exposure for normal animals, 10-day and 17-day tumor animals. (d-f) The corresponding $\mathrm{R}_{1}$ accumulation as a function of time over 80 minutes for (a)-(c).

first subgroup of experimental group 1 , the $R_{1}$-map signal of the BBB-opening area was increased from 0.1 to about 1.2 by FUS, and AUC maps showed an increase in accumulation of Gd-DTPA deposition from 20 to about 600 .

In tumor-bearing animals, the FUS-induced BBB area clearly covered the tumor tissue (Figure 4; small (10-day) or large (17-day) tumors). Sonication resulted in increased GdDTPA accumulation in the tumor and in the peripheral BBBopened area as evidenced by signal enhancement in the $\mathrm{R}_{1}$ map images. AUC maps showed maintained high staining intensities after Gd-DTPA injection. In the small (10-day) tumors, FUS resulted in an increase of $\mathrm{R}_{1}$ signal from 1 to about $1.5 \mathrm{~s}^{-1}$ and an increase in Gd-DTPA deposition of about 200 (from 400 to 600). However, in large (17-day) tumors, FUS did not lead to a significant change in the $\mathrm{R}_{1}$-signal, which increased from 1.9 to $2 \mathrm{~s}^{-1}$, or the AUC value which increased by only about 50 (from 1000 to 1050).

The kinetics of Gd-DTPA accumulation were evaluated after a single sonication treatment in thirty animals (normal rats: $n=18$; small-tumor model: $n=6$; large-tumor model: $n=6$ ). An ROI from the BBB-opened area on $\mathrm{T}_{2}$ weighted images (target) and the corresponding ROI from the contralateral brain (contralateral) were used to infer GdDTPA concentration from the $\mathrm{R}_{1}$ signals and the AUC over time in the 10-day tumors (volume $<0.05 \mathrm{~cm}^{3}$ ), 17-day tumors (volume $>0.05 \mathrm{~cm}^{3}$ ), or normal controls after sonication (Figure 5). Figures 5(a)-5(c) showed the comparison of changes in $R_{1}$ as a function of time (from 0 to $80 \mathrm{~min}$ ) for three typical animals. When considering the peak value over the whole scanning process, FUS caused the highest enhancement in $\mathrm{R}_{1}$ signal of contrast agent in normal tissue (from 0.2 to 0.84 ; Figure 5(a)). Sonication also led to a large increase in $R_{1}$ signal in 10-day tumor, from 0.86 to $1.48 \mathrm{~s}^{-1}$ (Figure 5(b)). However, the already high permeability of $17-$ day tumor to Gd-DTPA was not significantly increased by FUS, from 1.49 to $1.62 \mathrm{~s}^{-1}$ (Figure 5(c)).

The corresponding AUC (accumulation of $\mathrm{R}_{1}$ ) as a function of time (from 0 to $80 \mathrm{~min}$ ) in these three animals is shown in Figures 5(d)-5(f). In the normal animal, total GdDTPA accumulation in the BBB-opening area was increased from 44.2 to $552.6 \mathrm{pmol}$ by FUS (Figure 5(d)). Gd-DTPA accumulation in the 10-day tumor increased from 411.7 to 577.5 pmol, compared to only about 73 pmol over time in the contralateral control hemisphere (Figure 5(e)). However, 


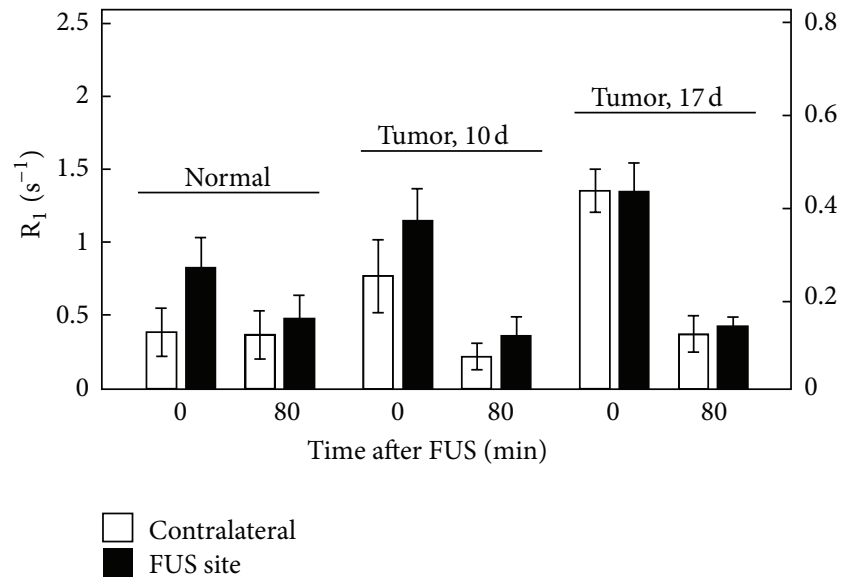

(a)

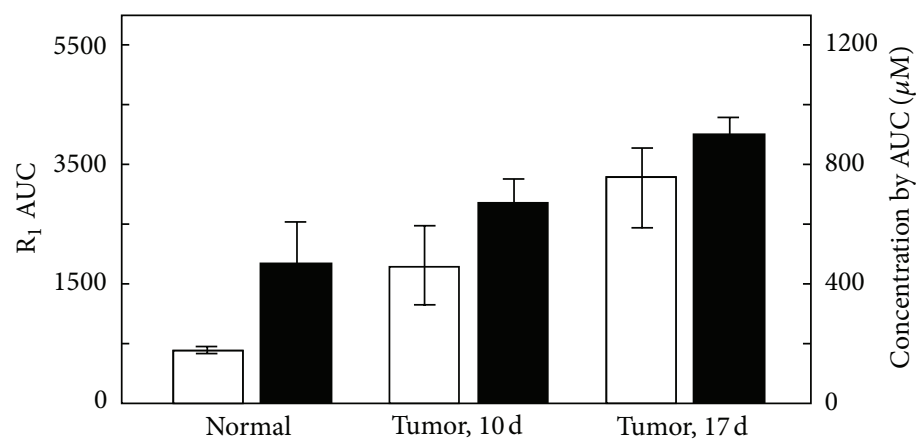

Contralateral

FUS site

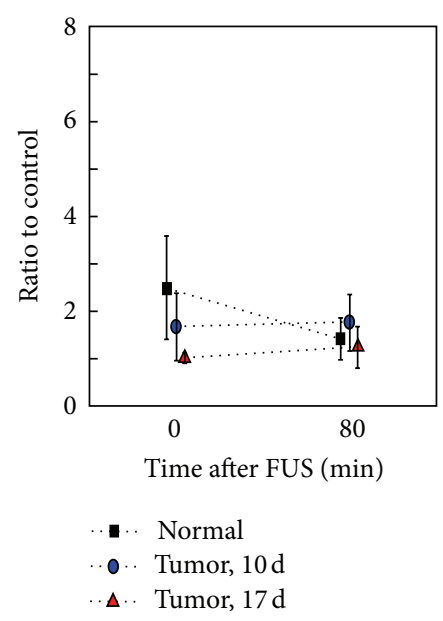

(b)

FIGURE 6: (a) Instantaneous $\mathrm{R}_{1}$ measured immediately or 80 minutes after FUS exposure in normal animals, 10-day and 17-day tumor animals. (b) Ratio of instantaneous $\mathrm{R}_{1}$ values, immediately and 80 min after FUS. (c) Corresponding $\mathrm{R}_{1} \mathrm{AUC}$ of (a). Images were acquired in time slot II.

Gd-DTPA accumulated to about the same high value with and without FUS ( 863.8 versus $867.3 \mathrm{pmol}$ ) in the 17 -day tumor (Figure 5(f)).

Gd-DTPA levels on the FUS-treated and contralateral side were also evaluated at either $10 \mathrm{~min}$ or $80 \mathrm{~min}$ after a single sonication treatment (Figure 6). The Gd-DTPA signal intensity increased in the BBB-opening area $10 \mathrm{~min}$ after sonication as evidenced by an increase in the $\mathrm{R}_{1}$ signal from 0.3863 to $0.8313 \mathrm{~s}^{-1}$ for control and from 0.77 to $1.15 \mathrm{~s}^{-1}$ for small-tumor animals. However, the contrast agent signals in the FUS-enhanced large tumors were the same as for the contralateral region (about $0.136 \mathrm{~s}^{-1}$ ). All $\mathrm{R}_{1}$ signals in these brain tissues returned to baseline (about $0.428 \mathrm{~s}^{-1}$ ) at 80 min after sonication. The ratio of $\mathrm{R}_{1}$ between the FUSexposed and control areas went from $2.48 \mathrm{~s}^{-1}$ at $10 \mathrm{~min}$ to $1.4 \mathrm{~s}^{-1}$ at $80 \mathrm{~min}$ in normal tissue and from about 1.8 to $1 \mathrm{~s}^{-1}$ for the tumor-bearing animals (Figure 6(b)). The AUC at $10 \mathrm{~min}$ and $80 \mathrm{~min}$ was compared between control and $\mathrm{BBB}$-opened brain regions. At $10 \mathrm{~min}$ after sonication, the accumulated Gd-DTPA concentration of the BBB-opened area increased to $465.99 \mu \mathrm{M}$, compared to $667.34 \mu \mathrm{M}$ for the small tumor and a limited increase to $896 \mu \mathrm{M}$ for the large tumor (Figure 6(c)).

Next we evaluated the correlation between EB leakage (Figure 7(a)) and Gd-DTPA accumulation (estimated by the AUC) in the same region of the brain. We found that the accumulated distribution of Gd-DTPA (i.e., AUC) was highly correlated with the distribution of $\mathrm{EB}\left(r^{2}=0.8897\right)$ (Figure $7(\mathrm{~b})$ ). Thus, $\mathrm{R}_{1}$-based pharmacodynamic analysis provided a reasonable map of the permeability of the BBBdisrupted region to EB dye over time. EB dye and GdDTPA accumulation showed the same tendency of higher overall permeability in tissues of large tumors and less dependence on FUS treatment, as evidenced from the ratios of accumulation between contralateral and FUS-treated regions (Figure 7(c)).

Next, we analyzed Gd-DTPA deposition dynamics in experimental group 2 animals by AUC analysis for three individual time slots: (I) before FUS, (II) immediately after FUS, and (III) 2 hours after FUS. Gd-DTPA concentration 


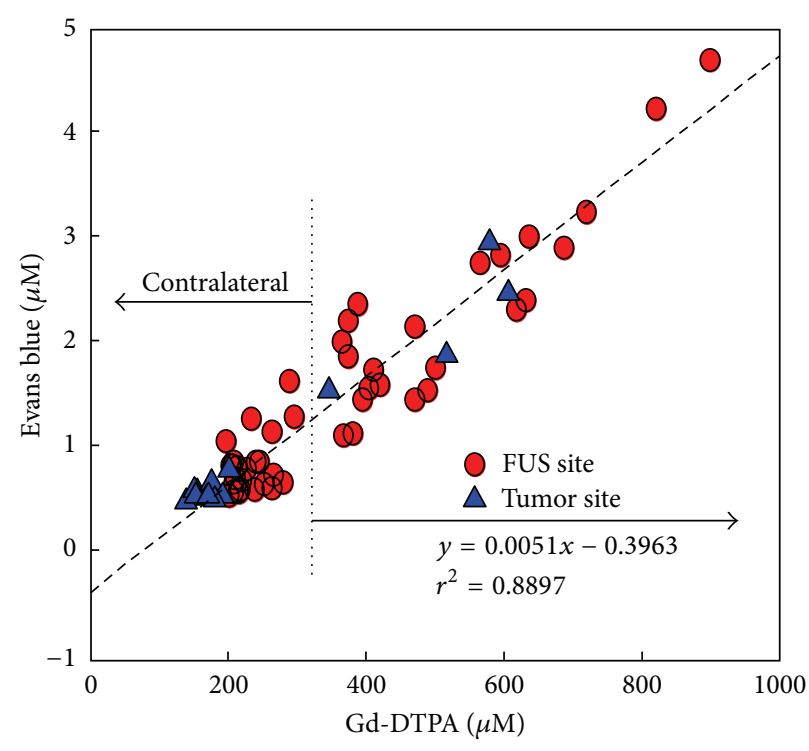

Normal BBB group

$\triangle$ Normal tumor group

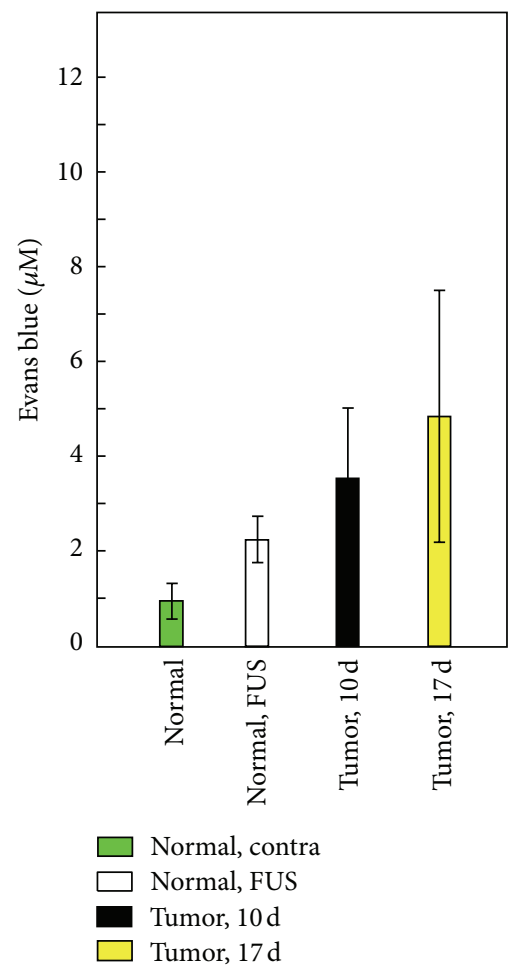

(b)

(a)

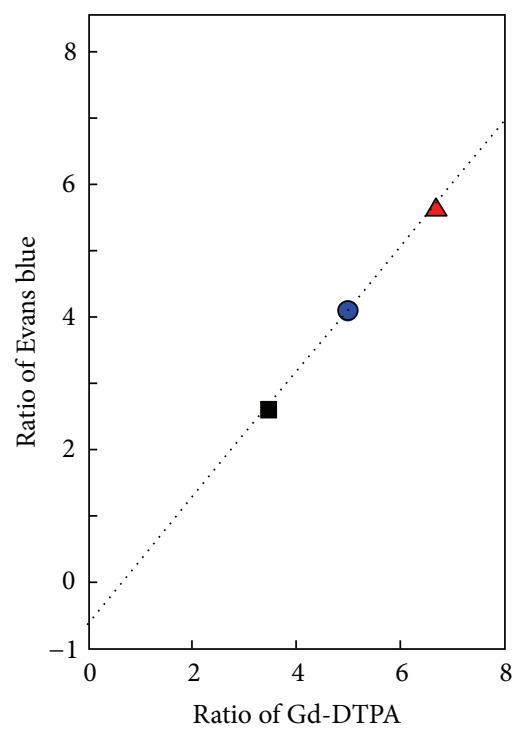

- Normal, FUS

Tumor, $10 \mathrm{~d}$

$\Delta$ Tumor, $17 \mathrm{~d}$

(c)

FIGURE 7: (a) Correlation between quantified Evans blue concentration and $\mathrm{R}_{1}$-estimated Gd-DTPA concentration in normal (red circle) and tumor (blue triangle) brains. Left arrow: contralateral side and right arrow: with FUS (red circle); normal tumor (blue triangle). (b) Evans blue after FUS exposure in the normal contralateral region, normal FUS targeting region, 10-day tumor region after FUS, and 17-day tumor region after FUS. (c) Correlation of the ratios of FUS: contralateral concentrations of Evans blue and Gd-DTPA in normal and tumor-bearing brains. 


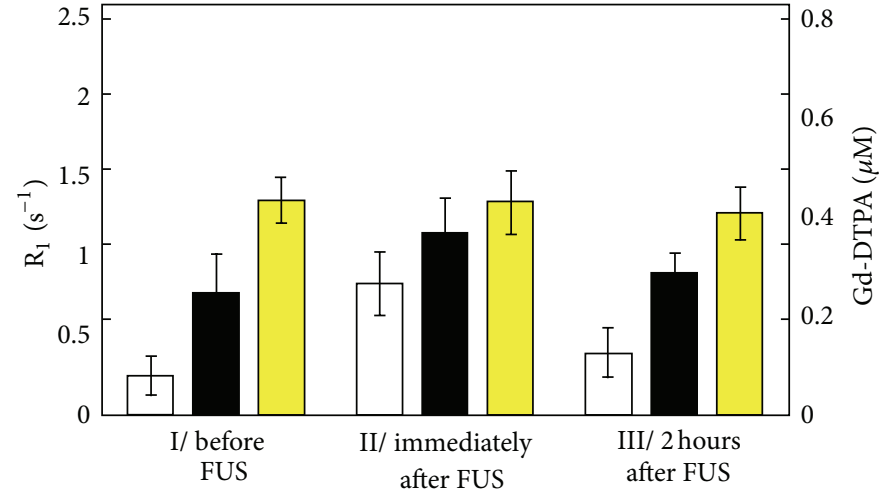

(a)

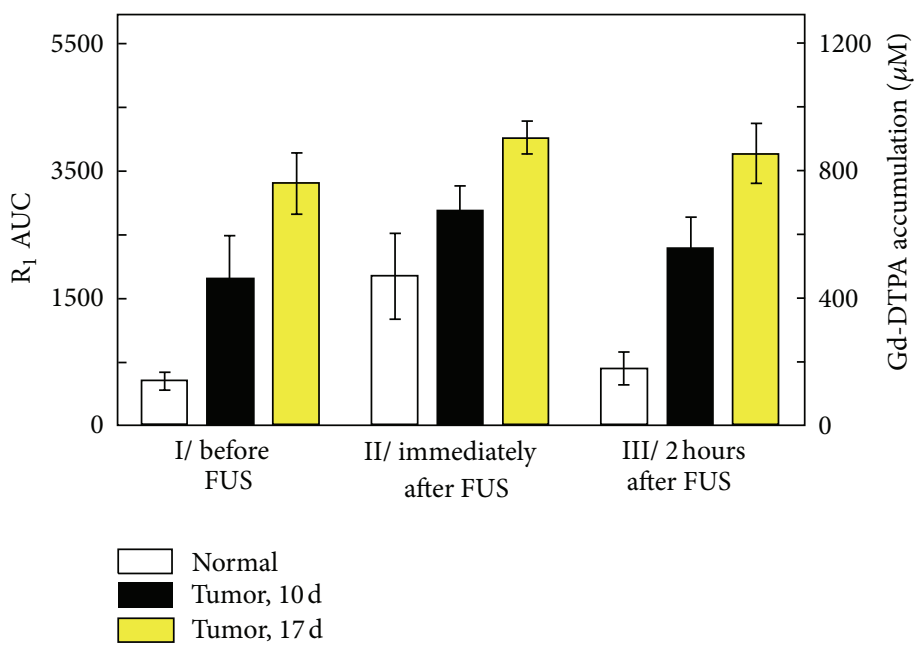

(c)

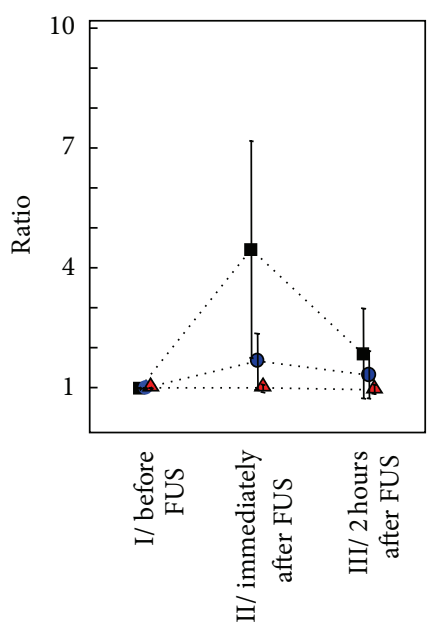

-. Normal

-. Small tumor

•.. Large tumor

(b)

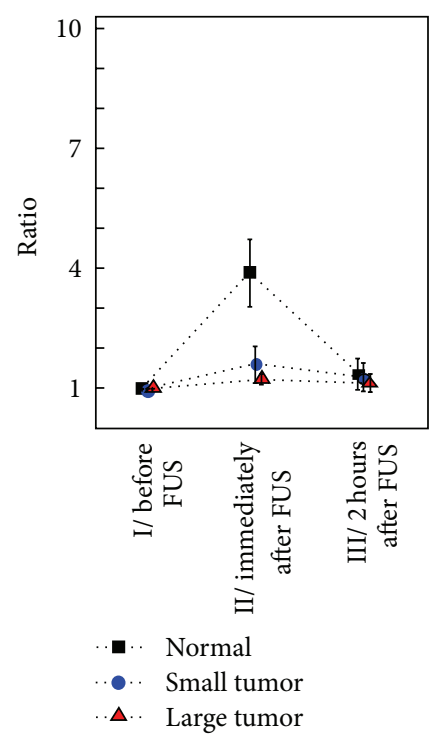

(d)

FIGURE 8: (a) Instantaneous $\mathrm{R}_{1}$ values of the normal animals, 10-day and 17-day tumor animals at MRI acquisition time slots I, II, and III. (b) Ratio of instantaneous $R_{1}$ to the value in time slot $I$. (c) Corresponding $R_{1}$ AUC of (a). (d) Ratio of $R_{1}$ AUC to the value in time slot $I$.

and accumulation in the target area presented the same trend at all three time points (Figure 8). As before, we observed a transient peak of $\mathrm{R}_{1}$ and the AUC in the BBB-opened brain just after sonication (Figures $8(\mathrm{a})$ and $8(\mathrm{c})$ ). However, the increase in the ratio of $R_{1}$ or AUC again differed between the normal and tumor-bearing animals (Figures $8(\mathrm{~b})$ and $8(\mathrm{~d})$ ). The ratio was highest in the normal BBB-opening area $\left(R_{1}\right.$ signal 4.4 times than before FUS; AUC: 3.7 times), followed by the small-tumor model $\left(\mathrm{R}_{1}\right.$ signal: 1.7 times; AUC: 1.6 times), with no significant change in the large tumor, confirming our previous observations that FUS did not significantly further affect permeability in large tumors. These ratios subsequently decreased at the time point 2 hours after FUS induction, returning to approximately the same values of DCE-MRI as originally observed before sonication. This result implied that at 2 hours, the BBB-opening area had recovered to the same baseline permeability level to contrast agent as prior to sonication.

HE staining of tumors 10 days after implantation showed even staining without scattered red blood cells in the absence of FUS (Figures 9(a) and 9(b)). Tumor cells were characterized by dense nuclear distribution, and only tiny areas of gliosis infiltrated with chronic inflammatory cells and some hemorrhaging were found (Figures 9 (c) and $9(\mathrm{~d})$ ). HE staining of tumors 17 days after implantation revealed a number of regions with extensive apoptosis and cavities in the core of the tumor, and hemorrhagic structures with scattered and spreading erythrocytes could be observed 


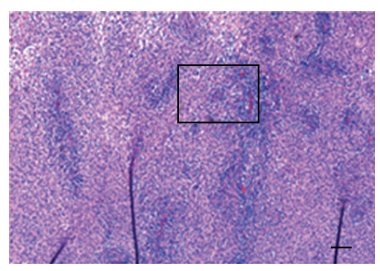

(a)

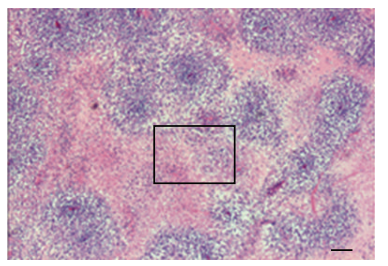

(e)

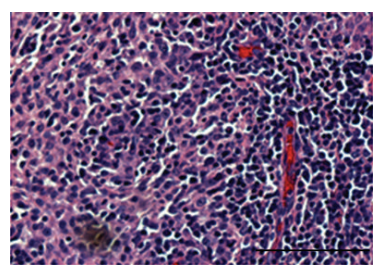

(b)

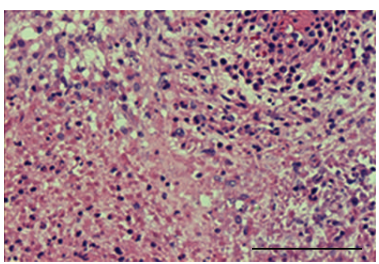

(f)

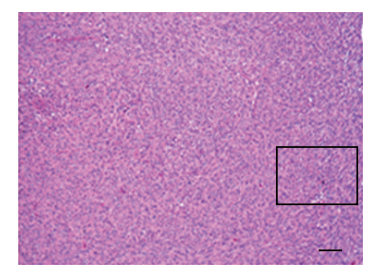

(c)

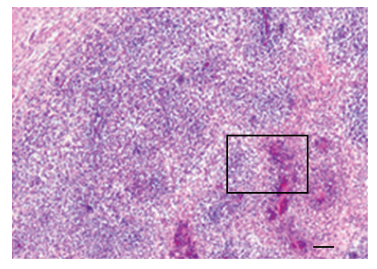

(g)

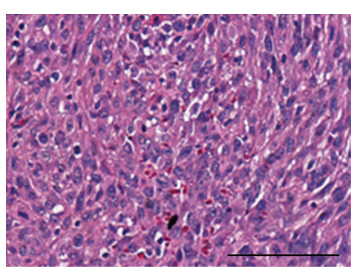

(d)

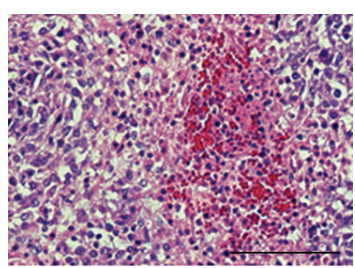

$100 \mu \mathrm{m}$

FIGURE 9: HE staining. (a) and (b) Small (10-day) tumor tissue without FUS exposure, 40x and 200x. (c) and (d) Small (10-day) tumor tissue with FUS exposure, 40x and 200x. (e) and (f) Large (17-day) tumor tissue without FUS exposure, 40x and 200x. (c) and (d) Large (17-day) tumor tissue with FUS exposure, 40x and 200x.
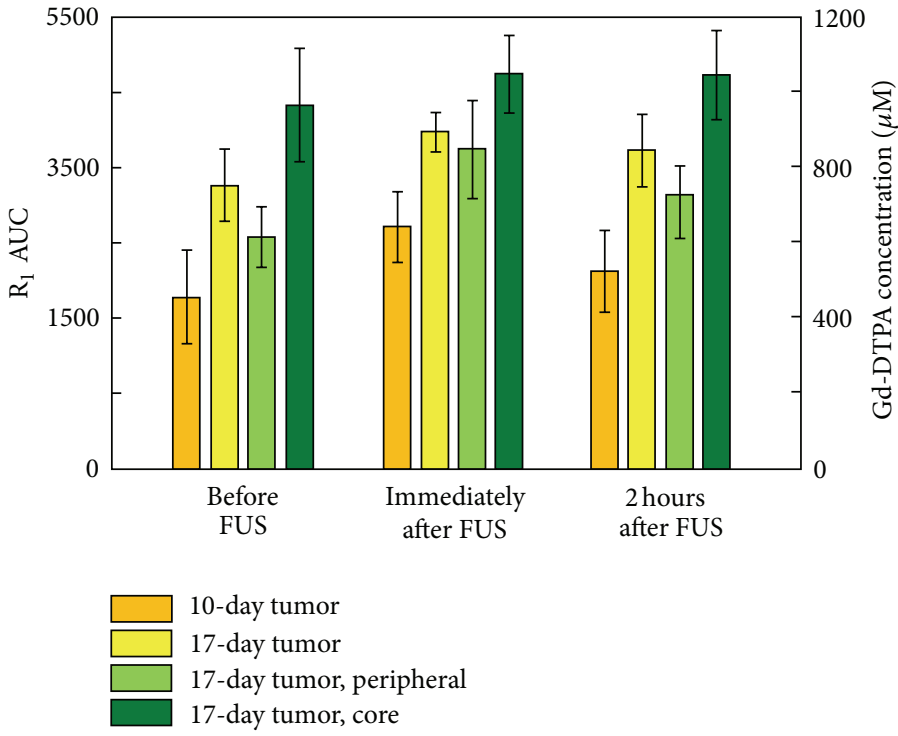

(a)
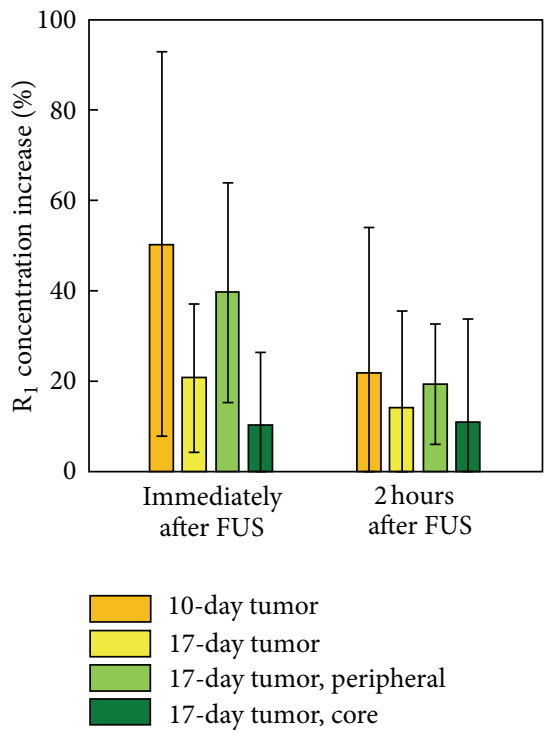

(b)

FIgURE 10: (a) $\mathrm{R}_{1}$ AUC analysis of the core and peripheral subregions of 17-day tumor. (b) $\mathrm{R}_{1}$ AUC increase relative to time slot $\mathrm{I}$.

around discontinuous vasculature (Figures $9(\mathrm{e})$ and $9(\mathrm{f})$ ), supporting our findings of high permeability of 17-day tumors based on observation of Gd-DTPA deposition. This structure did not change significantly after sonication (Figures $9(\mathrm{~g})$ and 9(h)) with hemorrhagic regions remaining similar to those in the unexposed tumor.

Previous reports showed that the tumor core consists of a bulky necrotic mass without functional vasculature, whereas the tumor periphery maintains a high degree of vasculature structure $[28,29]$. We therefore hypothesized that microbubble-enhanced FUS exposure would have a bigger effect on enhancing permeability in the peripheral tumor. We further divided the 17-day tumor animals into core and peripheral subregions and then repeated the MRI AUC analysis (Figure 10). We observed that, after FUS exposure, the 17-day peripheral tumor showed a similar trend to the 10day tumor, which showed a nearly 1.7 -fold of instantaneous increase and 50\% accumulation increase in Gd-DTPA, and the permeability dropped significantly two hours after FUS exposure to about half (20\%), which is similar to 10 -day tumor. In contrast, the 17-day tumor core mimicked the behavior of the undivided 17-day tumor, accumulating high 
levels of Gd-DTPA both before and after FUS exposure (AUC increase of only 10\% after FUS exposure) (Figure 10). This relatively low increase in the AUC persisted when the tumor core was re-evaluated at 2 hours after FUS exposure (9\%). These observations suggest that the FUS-BBB opening can provide the most pronounced drug delivery enhancing effect on tumor peripheral or tumors with high-vascularity stage tumors, yet only provides limited effects on bulky and necrotic tumors.

\section{Discussion}

This study demonstrated the pharmacodynamic characteristics of small-molecule leakage at various stages of tumors after application of microbubble-enhanced FUS to open the BBB. We analyzed two small molecules with similar molecular weights to obtain complimentary data on pharmacodynamic behavior. Gd-DTPA was used to provide contrast in MRI and for semiquantitative verification of biodistribution in vivo, and EB dye was used as a measure of drug accumulation after animal sacrifice. These two molecules, which normally do not enter the brain parenchyma from the bloodstream, could potentially be used as surrogate markers for drug delivery. Although the dynamic distribution of Gd-DTPA may differ from that of Evans blue, we demonstrated that the AUC accumulation of Gd-DTPA analyzed by MRI was highly correlated with EB accumulation in the brain $\left(r^{2}=0.8897\right)$, implying that MRI AUC analysis of Gd-DTPA could predict the concentration of EB accumulating in the brain, and may thus have the potential to predict the pharmacodynamic behavior and biodistribution of other therapeutic agents.

This study employed high-temporal-resolution dynamic CE-MRI that could be utilized for small-molecule in vivo distribution and semiquantification, as attempted in previous studies [30, 31]. The unique features provided by dynamic CE-MRI include the capability of rapid evaluation and high spatial resolution, as well as kinetic analysis to evaluate tumor perfusion [30]. Positron emission tomography (PET) has also been used for pharmacological studies in several tumor types [32, 33]. However, potential limitations of PET may include its limited imaging resolution and complexity of radiotracer synthesis. In small tumors, partial volume effects may be significant if the tumor size is less than twice the resolution of the scanner [34]. MRI methods provide the advantage of having good spatial resolution equal to that of corresponding morphologic images. In addition, MRI is minimally invasive and poses little risk to patients. We used voxels of about $0.26 \times 0.26 \times 1 \mathrm{~mm}^{3}$ to construct images sufficient for small-animal analysis. On the other hand, PET relies on radiolabeled molecules that bind to receptors to allow absolute quantification by detection of isotopes. PET may also be limited by its high cost, limited availability of radiotracer, and the need for a cyclotron as well as onsite radiochemistry for radioisotope production [35]. PET involves comprehensive conjugation of radiotracers and specific tailor-made molecules limiting its general use for pharmacodynamic analysis. Although Gd-DTPA cannot be directly conjugated to therapeutic molecules, the detection of coadministered Gd-DTPA by CE-MRI is highly correlated with targeted molecules, providing an excellent tool for monitoring vasculature and evaluating tissue/tumor permeability at high temporal/spatial resolution, suggesting its continued usefulness for pharmacodynamic analysis of brain drug delivery.

Tumor tissues are known to have high permeability due to the presence of large endothelial cell gaps, incomplete basement membrane, and the relative lack of pericyte or smooth muscle association with endothelial cells [36, 37]. In addition, the network of vasculature in solid tumors is markedly different from the normal hierarchical branching patterns and contains leaky vessel structures. Variations in permeability are also associated with the tumor grade as well as various neoplastic effects that could disrupt the BBB [38]. In this study, we observed that tumors with different levels of progression showed different characteristics of blood-vessel permeability and small-molecule accumulation. We found that the $\mathrm{AUC}_{80 \mathrm{~min}}$ in small tumors was $452 \pm 122.5 \mu \mathrm{M}$, whereas in large tumors it reached $754 \pm 48.3 \mu \mathrm{M}$. Moreover, we confirmed that FUS-BBB opening provided a $50 \%$ enhancement of accumulation of Gd-DTPA in small brain tumors and a $40 \%$ enhancement at the large tumor periphery, implying that FUS-BBB opening is an effective approach to increase brain-tumor permeability and therefore enhance delivery of therapeutic molecules.

Histological examination by $\mathrm{HE}$ staining showed that smaller (10-day) tumors had well-ordered vasculature with fewer abnormal endothelial cell gaps (Figures 9(a) and 9(b)). The blood vessel density in small tumor was lower, resulting in less Gd-DTPA and EB accumulation. In contrast, 17day tumor tissues contained more large fenestrae (Figures 9(e) and 9(f)), consistent with previous pathological findings that high-grade brain tumors contain neovasculature and apoptotic tumor cells, leading to hyperpermeability [39]. These pathological changes are consistent with the increased Gd-DTPA and EB accumulation that we observed in 17-day tumor tissues before sonication.

Although $\mathrm{AUC}_{80 \text { min }}$ correlated well with the pharmacodynamic behavior of another small molecule (EB), Gd-DTPA accumulation can be very different even under the same FUS exposure conditions, for example, varying from 365.2 to $900 \mu \mathrm{M}$ (Figure 7(b)). These large variations were likely due to differences in skull thickness and angle of incidence between the FUS beam and the skull surface among the animals [40], and the presence of standing waves produced in the skull cavity that alter the peak pressure at the target position and thus the level of BBB opening [41]. Since FUS$\mathrm{BBB}$ opening may vary substantially, it is essential to perform an AUC analysis during CE-MRI to monitor small-molecule delivery into the brain for individual subjects and targets.

\section{Conclusion}

In this study, we characterized the dynamics of BBB opening in normal and tumor tissues using DCE-MRI with GdDTPA contrast agent, and related them to the concentrations of Evans Blue determined from tissues after sacrifice. The 
concentrations of the surrogate tracer (Gd-DTPA) and EB dye showed a strong linear correlation. With this dynamic information of tumor permeability, the pharmacodynamic model can be modified to eventually take into account parameters that affect drug delivery over time. Tumor peripheral or high-vascular tumor may have the most significant benefit on blood-brain or blood-tumor permeability increase, which gives critical information when intending to apply FUS for brain drug enhanced delivery. We hope to use such pharmacodynamic predictions along with FUS-induced BBB opening to develop a method for image-guided drug delivery that can estimate the amount of drug that will be delivered to tissues at each time point.

\section{Conflict of Interests}

The authors declare that there is no conflict of interests.

\section{Acknowledgments}

The authors thank the Molecular Imaging Center, Chang Gung Memorial Hospital. This work was supported by the National Science Council, Taiwan (Grant nos. 100-2321B-182-010, 100-2120-M-182A-001, and 101-2221-E-182-002MY3).

\section{References}

[1] K. Hynynen, N. McDannold, N. Vykhodtseva, and F. A. Jolesz, "Noninvasive MR imaging-guided focal opening of the bloodbrain barrier in rabbits," Radiology, vol. 220, no. 3, pp. 640-646, 2001.

[2] K. Hynynen, N. McDannold, N. A. Sheikov, F. A. Jolesz, and N. Vykhodtseva, "Local and reversible blood-brain barrier disruption by noninvasive focused ultrasound at frequencies suitable for trans-skull sonications," NeuroImage, vol. 24, no. 1, pp. 12-20, 2005.

[3] K. Hynynen, N. McDannold, N. Vykhodtseva et al., "Focal disruption of the blood-brain barrier due to $260-\mathrm{kHz}$ ultrasound bursts: a method for molecular imaging and targeted drug delivery," Journal of Neurosurgery, vol. 105, no. 3, pp. 445-454, 2006.

[4] M. Kinoshita, N. McDannold, F. A. Jolesz, and K. Hynynen, "Targeted delivery of antibodies through the blood-brain barrier by MRI-guided focused ultrasound," Biochemical and Biophysical Research Communications, vol. 340, no. 4, pp. 10851090, 2006.

[5] N. McDannold, N. Vykhodtseva, and K. Hynynen, "Effects of acoustic parameters and ultrasound contrast agent dose on focused-ultrasound induced blood-brain barrier disruption," Ultrasound in Medicine and Biology, vol. 34, no. 6, pp. 930-937, 2008.

[6] H. L. Liu, Y. Y. Wai, W. S. Chen et al., "Hemorrhage detection during focused-ultrasound induced blood-brain-barrier opening by using susceptibility-weighted magnetic resonance imaging," Ultrasound in Medicine and Biology, vol. 34, no. 4, pp. 598-606, 2008.

[7] H. L. Liu, P. H. Hsu, P. N. Chu et al., "Magnetic resonance imaging enhanced by superparamagnetic iron oxide particles: usefulness for distinguishing between focused ultrasoundinduced blood-brain barrier disruption and brain hemorrhage," Journal of Magnetic Resonance Imaging, vol. 29, no. 1, pp. 31-38, 2009.

[8] M. Kinoshita, N. McDannold, F. A. Jolesz, and K. Hynynen, "Noninvasive localized delivery of Herceptin to the mouse brain by MRI-guided focused ultrasound-induced blood-brain barrier disruption," Proceedings of the National Academy of Sciences of the United States of America, vol. 103, no. 31, pp. 1171911723, 2006.

[9] L. H. Treat, N. McDannold, N. Vykhodtseva, Y. Zhang, K. Tam, and K. Hynynen, "Targeted delivery of doxorubicin to the rat brain at therapeutic levels using MRI-guided focused ultrasound," International Journal of Cancer, vol. 121, no. 4, pp. 901-907, 2007.

[10] J. Mei, Y. Cheng, Y. Song et al., "Experimental study on targeted methotrexate delivery to the rabbit brain via magnetic resonance imaging-guided focused ultrasound," Journal of Ultrasound in Medicine, vol. 28, no. 7, pp. 871-880, 2009.

[11] H. L. Liu, M. Y. Hua, P. Y. Chen et al., "Blood-brain barrier disruption with focused ultrasound enhances delivery of chemotherapeutic drugs for glioblastoma treatment," Radiology, vol. 255, no. 2, pp. 415-425, 2010.

[12] W. M. Pardridge, "The blood-brain barrier: bottleneck in brain drug development," NeuroRx, vol. 2, no. 1, pp. 3-14, 2005.

[13] A. Jemal, R. Siegel, E. Ward et al., "Cancer statistics, 2006," CA: A Cancer Journal for Clinicians, vol. 56, no. 2, pp. 106-130, 2006.

[14] S. A. Grossman and J. F. Batara, "Current management of glioblastoma multiforme," Seminars in Oncology, vol. 31, no. 5, pp. 635-644, 2004.

[15] L. H. Treat, N. McDannold, Y. Zhang, N. Vykhodtseva, and K. Hynynen, "Improved anti-tumor effect of liposomal doxorubicin after targeted blood-brain barrier disruption by MRI-guided focused ultrasound in rat glioma," Ultrasound in Medicine and Biology, vol. 38, no. 10, pp. 1716-1725, 2012.

[16] H. L. Liu, M. Y. Hua, H. W. Yang et al., "Magnetic resonance monitoring of focused ultrasound/magnetic nanoparticle targeting delivery of therapeutic agents to the brain," Proceedings of the National Academy of Sciences of the United States of America, vol. 107, no. 34, pp. 15205-15210, 2010.

[17] K. Liu, G. Wen, X. F. Lv et al., "MR imaging of cerebral extraventricular neurocytoma: a report of 9 cases," The American Journal of Neuroradiology, 2012.

[18] N. McDannold, N. Vykhodtseva, and K. Hynynen, "Bloodbrain barrier disruption induced by focused ultrasound and circulating preformed microbubbles appears to be characterized by the mechanical index," Ultrasound in Medicine and Biology, vol. 34, no. 5, pp. 834-840, 2008.

[19] N. Vykhodtseva, N. McDannold, and K. Hynynen, "Progress and problems in the application of focused ultrasound for blood-brain barrier disruption," Ultrasonics, vol. 48, no. 4, pp. 279-296, 2008.

[20] F. Y. Yang, W. M. Fu, W. S. Chen, W. L. Yeh, and W. L. Lin, "Quantitative evaluation of the use of microbubbles with transcranial focused ultrasound on blood-brain-barrier disruption," Ultrasonics Sonochemistry, vol. 15, no. 4, pp. 636-643, 2008.

[21] F. Y. Yang, W. M. Fu, R. S. Yang, H. C. Liou, K. H. Kang, and W. L. Lin, "Quantitative evaluation of focused ultrasound with a contrast agent on blood-brain barrier disruption," Ultrasound in Medicine and Biology, vol. 33, no. 9, pp. 1421-1427, 2007.

[22] J. J. Choi, J. A. Feshitan, B. Baseri et al., "Microbubble-size dependence of focused ultrasound-induced bloodBrain barrier 
opening in mice in vivo," IEEE Transactions on Biomedical Engineering, vol. 57, no. 1, pp. 145-154, 2010.

[23] R. Chopra, N. Vykhodtseva, and K. Hynynen, "Influence of exposure time and pressure amplitude on blood-brain-barrier opening using transcranial ultrasound exposures," ACS Chemical Neuroscience, vol. 1, no. 5, pp. 391-398, 2010.

[24] J. Park, Y. Zhang, N. Vykhodtseva, F. A. Jolesz, and N. J. McDannold, "The kinetics of blood brain barrier permeability and targeted doxorubicin delivery into brain induced by focused ultrasound," Journal of Controlled Release, vol. 162, no. 1, pp.134142, 2012.

[25] P. S. Tofts and A. G. Kermode, "Measurement of the bloodbrain barrier permeability and leakage space using dynamic MR imaging. 1. Fundamental concepts," Magnetic Resonance in Medicine, vol. 17, no. 2, pp. 357-367, 1991.

[26] F. Vlachos, Y. S. Tung, and E. Konofagou, "Permeability dependence study of the focused ultrasound-induced bloodbrain barrier opening at distinct pressures and microbubble diameters using DCE-MRI," Magnetic Resonance in Medicine, vol. 66, no. 3, pp. 821-830, 2011.

[27] I. M. Noebauer-Huhmann, P. Szomolanyi, V. Juras, O. Kraff, M. E. Ladd, and S. Trattnig, "Gadolinium-based magnetic resonance contrast agents at 7 tesla: in vitro T1 relaxivities in human blood plasma," Investigative Radiology, vol. 45, no. 9, pp. 554-558, 2010.

[28] E. A. Neuwelt, P. A. Barnett, C. I. McCormick, L. G. Remsen, R. A. Kroll, and G. Sexton, "Differential permeability of a human brain tumor xenograft in the nude rat: impact of tumor size and method of administration on optimizing delivery of biologically diverse agents," Clinical Cancer Research, vol. 4, no. 6, pp. 15491555, 1998.

[29] H. Sarin, A. S. Kanevsky, H. Wu et al., "Effective transvascular delivery of nanoparticles across the blood-brain tumor barrier into malignant glioma cells," Journal of Translational Medicine, vol. 6, article 80, 2008.

[30] A. R. Padhani and J. E. Husband, "Dynamic contrast-enhanced MRI studies in oncology with an emphasis on quantification, validation and human studies," Clinical Radiology, vol. 56, no. 8, pp. 607-620, 2001.

[31] P. S. Tofts, G. Brix, D. L. Buckley et al., "Estimating kinetic parameters from dynamic contrast-enhanced T(1)-weighted MRI of a diffusable tracer: standardized quantities and symbols," Journal of Magnetic Resonance Imaging, vol. 10, no. 3, pp. 223-232, 1999.

[32] H. Olsson, C. Halldin, C. G. Swahn, and L. Farde, "Quantification of [11C]FLB 457 binding to extrastriatal dopamine receptors in the human brain," Journal of Cerebral Blood Flow and Metabolism, vol. 19, no. 10, pp. 1164-1173, 1999.

[33] K. W. Kang, D. S. Lee, J. H. Cho et al., "Quantification of F18 FDG PET images in temporal lobe epilepsy patients using probabilistic brain atlas," NeuroImage, vol. 14, no. 1, part 1, pp. $1-6,2001$.

[34] S. L. Bacharach, S. K. Libutti, and J. A. Carrasquillo, "Measuring tumor blood flow with $\mathrm{H}_{2}{ }^{15} \mathrm{O}$ : practical considerations," Nuclear Medicine and Biology, vol. 27, no. 7, pp. 671-676, 2000.

[35] M. S. Alavijeh and A. M. Palmer, "Measurement of the pharmacokinetics and pharmacodynamics of neuroactive compounds," Neurobiology of Disease, vol. 37, no. 1, pp. 38-47, 2010.

[36] H. F. Dvorak, L. F. Brown, M. Detmar, and A. M. Dvorak, "Vascular permeability factor/vascular endothelial growth factor, microvascular hyperpermeability, and angiogenesis," The
American Journal of Pathology, vol. 146, no. 5, pp. 1029-1039, 1995.

[37] L. E. Benjamin, D. Golijanin, A. Itin, D. Pode, and E. Keshet, "Selective ablation of immature blood vessels in established human tumors follows vascular endothelial growth factor withdrawal," Journal of Clinical Investigation, vol. 103, no. 2, pp. 159165, 1999.

[38] J. S. Taylor, P. S. Tofts, R. Port et al., "MR imaging of tumor microcirculation: promise for the new millennium," Journal of Magnetic Resonance Imaging, vol. 10, no. 6, pp. 903-907, 1999.

[39] L. F. Brown, K. T. Yeo, B. Berse et al., "Expression of vascular permeability factor (vascular endothelial growth factor) by epidermal keratinocytes during wound healing," Journal of Experimental Medicine, vol. 176, no. 5, pp. 1375-1379, 1992.

[40] M. A. O’Reilly, A. Muller, and K. Hynynen, "Ultrasound insertion loss of rat parietal bone appears to be proportional to animal mass at submegahertz frequencies," Ultrasound in Medicine and Biology, vol. 37, no. 11, pp. 1930-1937, 2011.

[41] M. A. O’Reilly, Y. Huang, and K. Hynynen, “The impact of standing wave effects on transcranial focused ultrasound disruption of the blood-brain barrier in a rat model," Physics in Medicine and Biology, vol. 55, no. 18, pp. 5251-5267, 2010. 


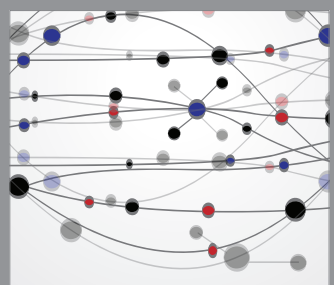

The Scientific World Journal
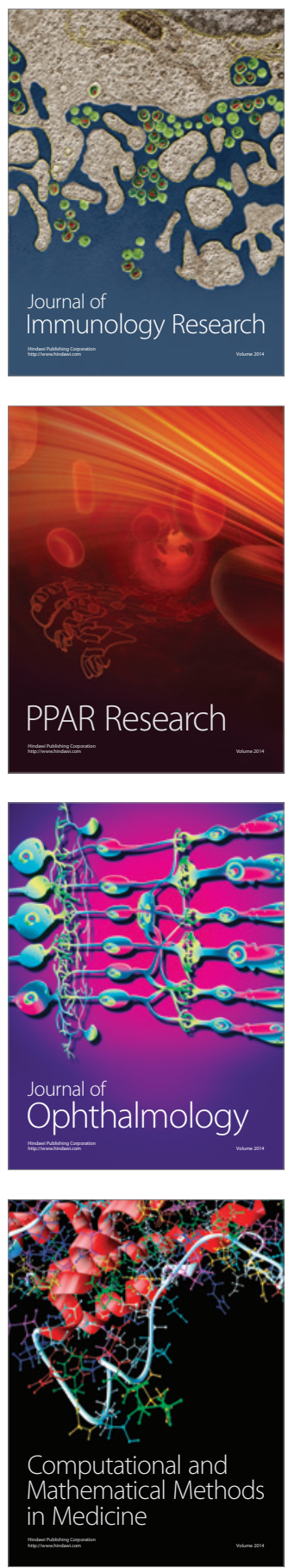

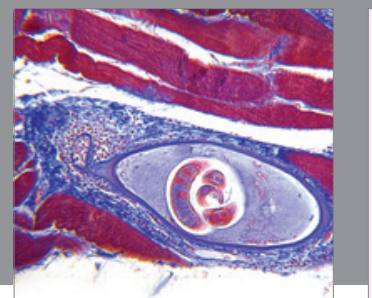

Gastroenterology

Research and Practice
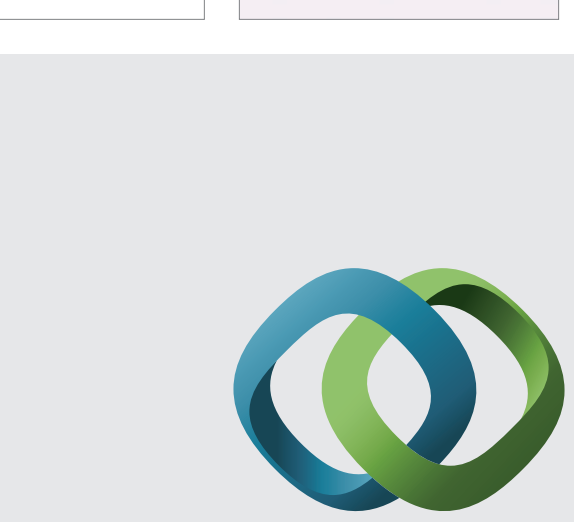

\section{Hindawi}

Submit your manuscripts at

http://www.hindawi.com
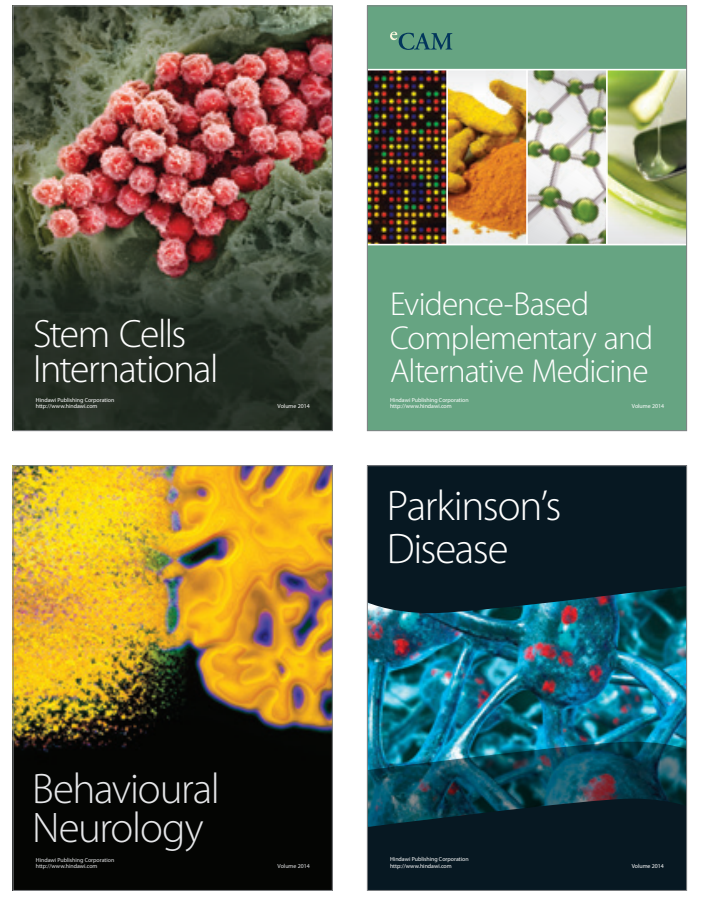
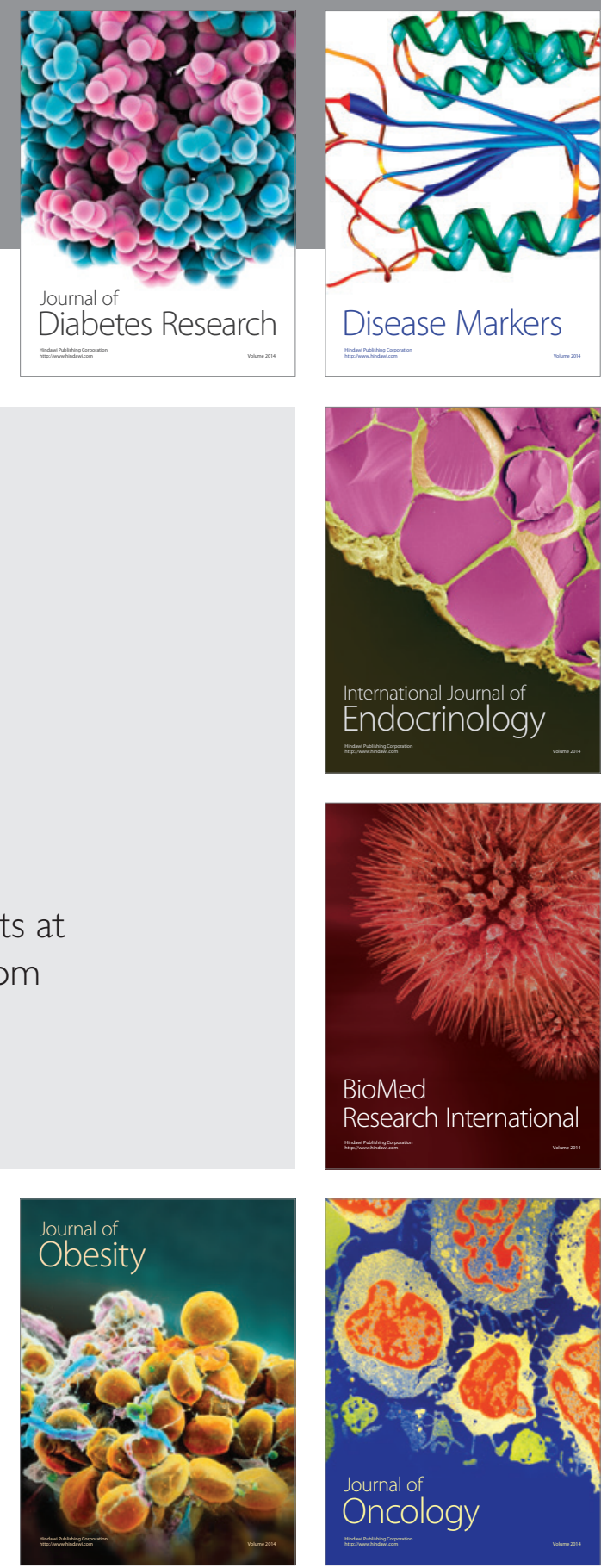

Disease Markers
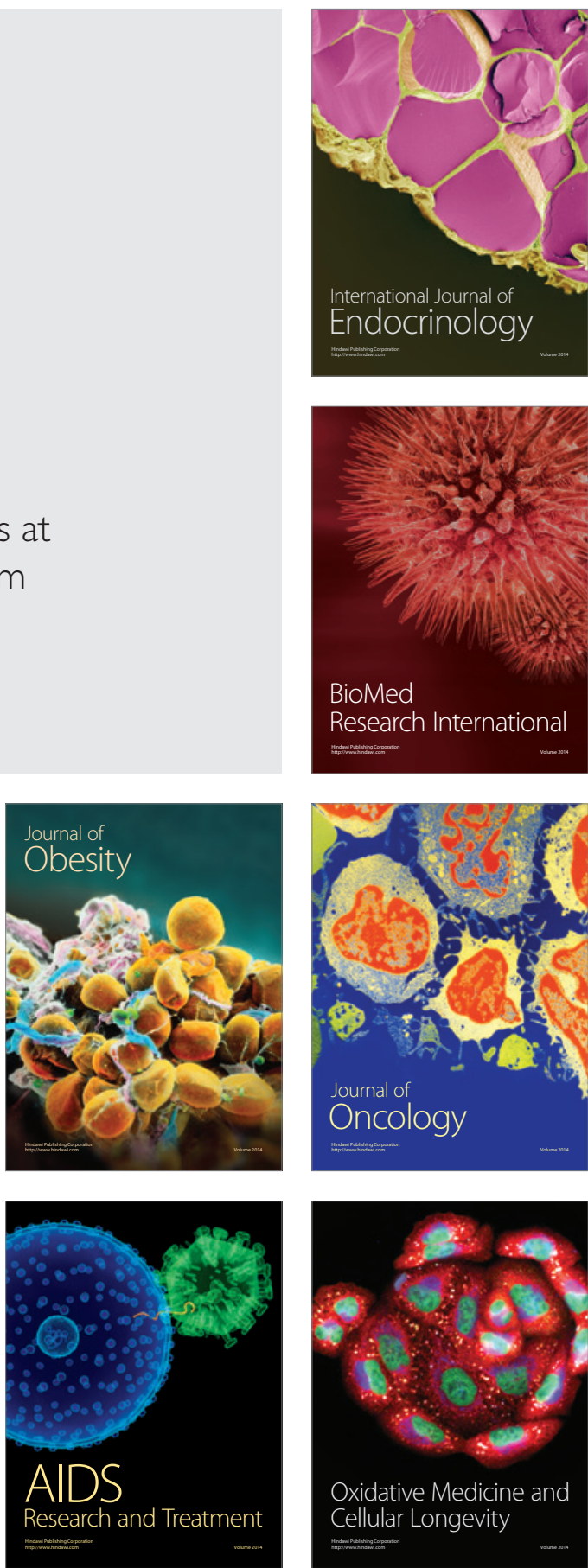